\title{
Comparative Study of the Gas-phase Cyclodimer Formations of Uracil and 6-azauracil in Excited State and through Conical Intersections $S_{0} / S_{1}$
}

\author{
Pavlina Bogomilova Kancheva ${ }^{\star}$ and Vassil Borissov Delchev \\ University of Plovdiv Paisii Hilendarski, Dept. Physical Chemistry, 24 Tzar Asen, 4000 Plovdiv, Bulgaria \\ *Corresponding author: E-mail: pkancheva@uni-plovdiv.net \\ Tel.: 00359896545727
}

Received: 28-11-2017

\begin{abstract}
The photocyclodimerization mechanisms of two oxo tautomers of uracil and 6-azauracil were studied at the CC2 level of theory and cc-pVDZ basis functions. Uracil was explored in three orientations of the monomers - cis-anti, trans-syn and trans-anti, while 6-azauracil - in all four orientations. Conical intersections $\mathrm{S}_{0} / \mathrm{S}_{1}$ were found at the $\operatorname{CASSCF}(2,2) / 6-31 \mathrm{G}^{*}$ theoretical level. The study propose a photophysical mechanisms of photoformation and photodestruction of cyclodimers (CDs), which occur through the ${ }^{1} \pi \pi^{*}$ electron excited states (spectroscopically bright states). The photophysical relaxations (internal conversions) to the ground states are mediated by conical intersections $S_{0} / S_{1}$, which we found and discussed in the current paper.
\end{abstract}

Keywords: $A b$ initio calculations; CASSCF optimizations; Conical intersections $\mathrm{S}_{0} / \mathrm{S}_{1}$; Pyrimidine cyclodimers; Uracil; 6-azauracil

\section{Introduction}

The photostability of nucleobases has a fundamental role in the protection of living organisms from the damaging UV light. ${ }^{1}$ The nucleic acid bases are main UV chromophores in cells. They absorb UV radiation under 300 $\mathrm{nm}$ and participate in a number of photoreactions - in particular internal conversions to ground states. These processes are connected with ring deformations and they are responsible for the photostability of nucleobases. ${ }^{2,3}$ The competitive $[2+2]$ cyclodimerizations through excited states are the second most common response of the pyrimidine nucleobases exposed to UV irradiation. ${ }^{2,4}$

The cyclodimers (CDs), formed by two adjacent pyrimidine bases are considered to be the most prevailing lesions of cellular DNA induced by the UV radiation. They can cause death of cells and skin cancer. ${ }^{4-11}$ The yield of formation of the CDs with a four-membered cyclobutane ring is larger than other photoadducts. ${ }^{712-17}$ The recognition and the repair of such DNA lesions is possible through different enzyme pathways. For example, the DNA photolyase catalyzes the cleavage of the cyclobutane bonds formed between the monomers. Thus the pyrimidine CD turns back in the initial stacked dimer. ${ }^{18,19}$
The found possible orientations of pairs of pyrimidines to form CD are four: cis-syn, trans-syn, cis-anti and trans-anti. Such structures are shown in Fig. 1. ${ }^{20}$

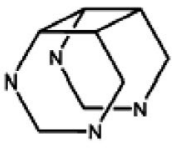

Cis-syn

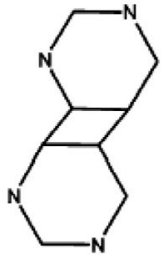

Cis-anti

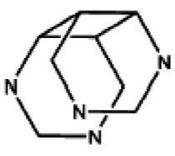

Trans-syn

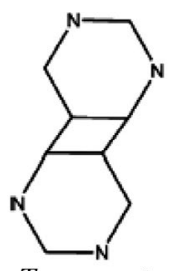

Trans-anti
Fig. 1. Four CDs of pyrimidine nucleobases

After irradiation of frozen water solution of uracil (U) the cis-syn and cis-anti CDs are predominantly formed. ${ }^{21-23}$ The trans-syn and trans-anti isomers are also present but in significantly lower amounts. ${ }^{23}$ The analysis (NMR, chromatography, etc.) has shown that the cis-syn $\mathrm{CD}$ is the major photoproduct. ${ }^{21-24}$ All four CDs are formed after irradiation of a fluid solution (water or acetonitrile) and when the aqueous solution is acetone sensitized. ${ }^{23-26}$ The trans-syn cyclodimers of $\mathrm{U}$ can be formed 
also by irradiation of denaturated DNA. ${ }^{27}$ The molecular and crystal structure of cis-syn photodimer of $\mathrm{U}$ has been proposed by Adman. ${ }^{24}$

6-Azauracil (6-AU) is a close analogue to U. The compound has an extra nitrogen atom in the aromatic ring. With respect to the biological effects, 6 -AU has been shown to inhibit animal tumors, ${ }^{28}$ human acute leuke$\mathrm{mia}^{29}$ and growth of a large number of microorganisms $^{30,31}$. The compound is also used as an antiviral drug. ${ }^{32}$ The IR absorption spectrum of 6-AU in low temperature matrix has shown that the diketo tautomer is predominant. ${ }^{33}$ In the UV absorption spectra of 6-AU in acetonitrile; two peaks are registered - at $200 \mathrm{~nm}$ and $260 \mathrm{~nm} .^{34}$ The last one has been assigned to a $\pi \rightarrow \pi^{*}$ electron transition as in U. Some authors claimed that the main relaxation process of $6-\mathrm{AU}$ is the intersystem crossing (ISC) mechanism involving a triplet state $\left({ }^{3} \pi \pi^{*}\right) \cdot{ }^{34-36}$ Irradiation with a wavelength of $308 \mathrm{~nm}$ has confirmed that the ISC process is predominant in 6-AU but it is accompanied with weak fluorescence. ${ }^{34}$

The mechanisms of formation of the cis-syn cyclophotodimer of thymine ${ }^{37-39}$ uracil $^{40-42}$ and cytosine ${ }^{43,44}$ have been proposed also by theoretical computations. It has been concluded that these processes are ultrafast and barrierless photoreactions in which the driving state is the first ${ }^{1} \pi \pi^{*}$ excited state which relaxes through a conical intersection (CI) $\mathrm{S}_{0} / \mathrm{S}_{1} \cdot{ }^{45}$ The triplet states have been also suggested to play a role in the photodimerizations of pyrimidine bases. ${ }^{12,46,47}$ It has been found that upon $266 \mathrm{~nm}$ radiation the CDs are formed via the singlet channel which is 30 times more efficient than the triplet one. By increasing the irradiation wavelength, the importance of the singlet channel is also increased. ${ }^{48}$

The aim of this study is to investigate theoretically the two close analogues $\mathrm{U}$ and 6-AU in order of their ability to form CDs through the bright ${ }^{1} \pi \pi^{*}$ excited states in the gas phase. It is interesting to see whether the extra nitrogen atom in the ring of 6-AU as compared to $\mathrm{U}$ would influence the photophysics of the compound.

\section{Theoretical Methods}

The structures of the stacked dimers (SDs), conical intersections $S_{0} / S_{1}$, and CDs of $U$ and 6-AU were optimized with the Complete-active-space self-consistent field (CASSCF) method and $6-31 \mathrm{G}^{*}$ basis functions. The active space was constructed by two electrons on two orbitals: HOMO and LUMO - they are shown in Fig. 2 (the remaining MOs are given in the electronic supplementary

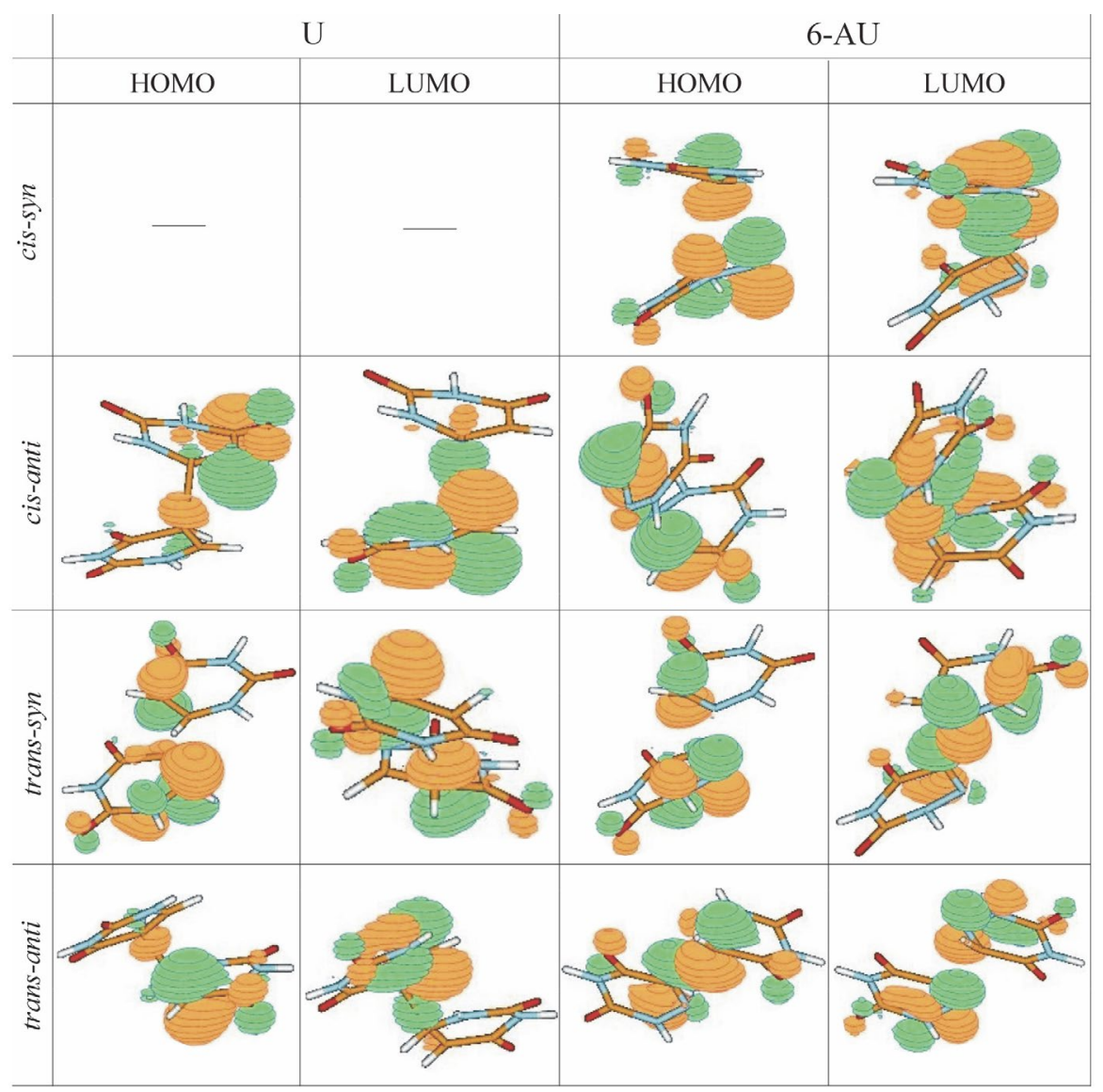

Fig. 2. HOMO and LUMO molecular orbitals of the conical intersections $S_{0} / S_{1}$ included in the active space $(2,2)$ 
information). Since the structures under study are rather complicated - including valent and non-valent bonds - we applied a limited active space for the optimizations. Our experience imposes this because the extension of the active space usually leads to serious troubles with the configuration wavefunction. - In other words a large active space includes a great many excited states and thus leads to a location of wrong conical intersections $S_{0} / S_{1}$. In order to be consistent with the level of computation, the branching space vectors (the gradient difference and derivative coupling vectors) were computed at the same theoretical level. Subsequently, we reoptimized the structures of the geometries-minima at the CC2 level (approximate second-order coupled-cluster method $)^{49}$ with cc-pVDZ basis functions. Additional computations of the CC2-vertical excitation energies of the CDs and SDs of $U$ and 6-AU were performed at this level either. No symmetry and geometry restrictions were applied for the optimizations of CBDs and the conical intersections $\mathrm{S}_{0} / \mathrm{S}_{1}$.

All SDs were optimized by freezing of one or two distances between the monomers forming the four-membered ring (see electronic supplementary information). The freezing of one of the distances is required since the structures lie in very shallow minima on the hyperPESs and the fully relaxed optimizations lead to planar WatsonCrick dimers. To find the distance(s) for freezing we performed preliminary SCAN computations of the CDs with respect to the responsible distance(s). We located the minimal distance(s) at which only Van der Waals forces start to act between the two monomers.

The excited-state reaction paths connecting the SDs and the CDs through conical intersections $\mathrm{S}_{0} / \mathrm{S}_{1}$ were found by the linear-interpolation-in-internal-coordinates approach (LIIC). It was performed in two steps: 1) interpolation between the SD and the conical intersection $\mathrm{S}_{0} /$ $S_{1}$, and 2) interpolation between the conical intersection $\mathrm{S}_{0} / \mathrm{S}_{1}$ and the CD. The internal coordinates of the interpolated structures $\left(q_{i}\right)$ along the reaction paths were generated by equation (1):

$$
q_{i}=q_{r}+\varepsilon\left(q_{p}-q_{r}\right),
$$

where $q_{r}$ - internal coordinate of the reactant (one of the minima in this case); $q_{p}$ - internal coordinate of the product (the conical intersection $S_{0} / S_{1}$ in this case); $\varepsilon$ - interpolation parameter, which varies from 0 (at the reactant) to 1 (at the product).

In each LIIC-step several intermediate structures were generated and their CC2-excitation energies were computed for the construction of the excited-state reaction paths. The proposed mechanisms concern the reactions in the gas phase.

The geometry optimizations of the conical intersections $\mathrm{S}_{0} / \mathrm{S}_{1}$, the calculations of the branching space vectors, and the equilibrium structures of the minima were carried out with the GAUSSIAN 03 program package..$^{50}$ The reac- tion paths (CC2) were studied with the Turbomole program. ${ }^{51}$ The programs MOLDEN ${ }^{52}$ and $\mathrm{Chemcraft}^{53}$ were used for the visualization of the results.

\section{Results and Discussion}

The CASSCF equilibrium geometries of the CDs, SDs as well as the conical intersections $\mathrm{S}_{0} / \mathrm{S}_{1}$ of $\mathrm{U}$ and 6-AU are shown in Fig. 3. The frontier MO included in the active space (HOMO and LUMO) of the conical intersections $\mathrm{S}_{0} /$ $S_{1}$, depicted in Fig. 2 are of $\pi$-type. The typical $\pi \rightarrow \pi^{*}$ charge transfer states are those in cis-anti and trans-anti conical intersections $\mathrm{S}_{0} / \mathrm{S}_{1}$ of $\mathrm{U}$ and in cis-syn and trans-syn conical intersections $S_{0} / S_{1}$ of $6-A U$.

The aromatic rings of the CDs of $U$ are nonplanar. The nonplanarity is a result of the repulsion between the $\pi$-electron clouds of the rings and of the lone electron pairs of the oxygen atoms. The C-C' bonds between the monomers are about $1.55 \AA$ long. The rings have envelope conformations, mutually twisted one to another (see electronic supplementary information). In contrast to the $\mathrm{CDs}$, the SD structures of $\mathrm{U}$ have planar aromatic rings. In the anti- configurations, the $\mathrm{C}-\mathrm{C}^{\prime}$ distances have close lengths, whereas in the trans-syn structure they differ with about $0.62 \AA$. In the cis-syn structure, obtained by Domcke et al. ${ }^{38}$ the corresponding difference is about $0.16 \AA$. In the $\mathrm{SDs}$, the aromatic rings of the cis-anti $\mathrm{U}$ are slightly twisted one to another. In the trans-syn isomer, the rings are highly twisted which means that the formation of the cyclodimer includes a reorientation of the aromatic rings of the $\mathrm{SD}$ (see the electronic supplementary information). In the trans-anti SD, instead twisting of both rings there is a parallel translation of the monomers and they form a "sandwich"-like structure.

The CDs of 6-AU and $\mathrm{U}$ are with nonplanar aromatic rings and "envelope" conformations. In the syn-6-AU structures, the four-membered ring has one $\mathrm{N}-\mathrm{N}^{\prime}$ and one $\mathrm{C}-\mathrm{C}^{\prime}$ 'bond, which show difference about $0.075 \AA$ A. In the anti- orientations, these bonds are of $\mathrm{N}-\mathrm{C}^{\prime}\left(\right.$ or $\left.\mathrm{C}-\mathrm{N}^{\prime}\right)$ type and they are equal in length. The SDs of 6-AU are generally very similar to the corresponding structures of $U$. The aromatic rings of the syn-structures are highly twisted one to another, while the structure of trans-anti is a "sandwich"-like; analogous to U. The SDs of 6-AU are with almost planar aromatic rings, like those of $U$ and the single monomers.

\section{1. Conical Intersections $S_{0} / S_{1}$}

All conical intersections $S_{0} / S_{1}$ of $U$ (Fig. 3) are structurally closer to the cyclodimers than to the SDs and have nonplanar aromatic rings. Only the hydrogen atoms from the four-membered rings deviate considerably from the planarity. The $\mathrm{C}-\mathrm{C}^{\prime}$ distances between the two pyrimidine monomers are shorter than those of the SDs and differ in 

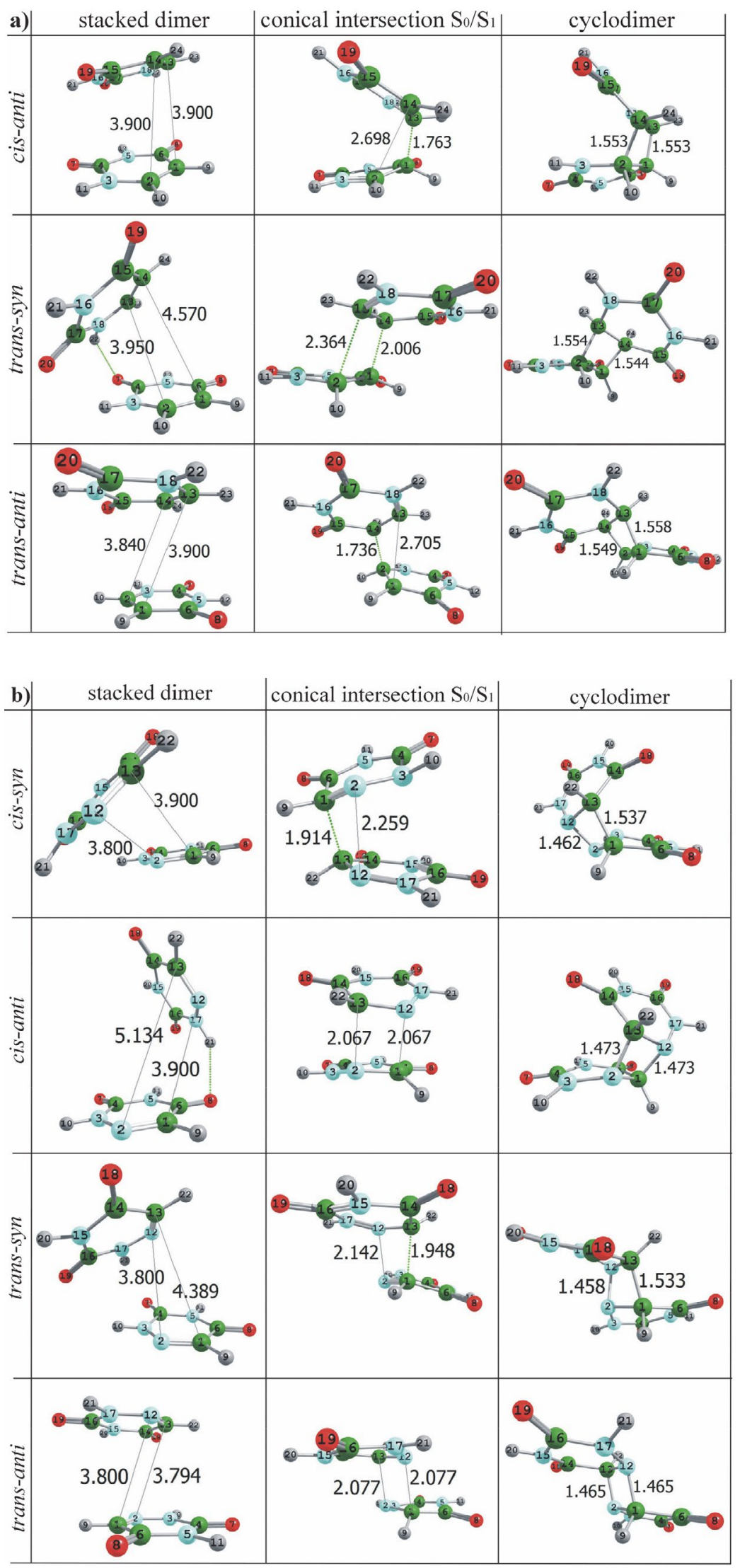

Fig. 3. CASSCF-optimized structures of CDs, conical intersections $S_{0} / S_{1}$ and $S D s$ of a) $U$ and b) 6-AU 
lengths. The anti- structures are with mutually twisted rings. The conical intersections $\mathrm{S}_{0} / \mathrm{S}_{1}$ of $\mathrm{U}$ exhibit a twisting of the ring $\mathrm{C}=\mathrm{C}$ bond, typical for the single pyrimidine conical intersections $S_{0} / S_{1}^{2,3,54-59}$ which have found to be the main channel for internal conversions in nucleobases.

All conical intersections $S_{0} / S_{1}$ of 6-AU have nonplanar aromatic rings, similar to those of $U$. The two closest distances between the two monomers depend on the orientation of the rings. In the syn-isomers we have different in length distances $\mathrm{C}-\mathrm{C}^{\prime}$ and $\mathrm{N}-\mathrm{N}$. In the anti-isomers these are the two $\mathrm{C}-\mathrm{N}^{\prime}$ distances which are equidistant. The conical intersections $\mathrm{S}_{0} / \mathrm{S}_{1}$ of $6-\mathrm{AU}$, like those of $\mathrm{U}$, are structurally closer to the cyclodimers than to the SDs. The conical intersections $\mathrm{S}_{0} / \mathrm{S}_{1}$ of 6 - $\mathrm{AU}$ are in general similar to those of $U$, but their aromatic rings are not as much twisted one to another as in $U$.

The calculated branching space vectors of the conical intersections $S_{0} / S_{1}$ are illustrated in the electronic supplementary information. They indicate clearly the trend of the systems to form four-membered rings. We believe that the found conical intersections $S_{0} / S_{1}$ mediate the photocyclodimerizations of $\mathrm{U}$ and 6-AU via internal conversions.

\section{2. Vertical Excitation Energies}

The CC2/cc-pVDZ vertical excitation energies of the systems SDs and CDs of the two compounds are listed in Table 1. One can see that for $\mathrm{U}$ the cis-and trans-anti $\mathrm{CDs}$ have higher vertical excitation energy of the bright ${ }^{1} \pi \pi^{*}$ excited states than the trans-syn CD. The first two excited states of all isomers of $U$ (for SDs and CDs) are the spectroscopically dark ${ }^{1} n \pi^{*}$ states.

Comparing the vertical excitation energies of the $\mathrm{CDs}$ of $\mathrm{U}$ with the UV absorption maximum of uracil in the gas phase $-4.79 \mathrm{eV}(259 \mathrm{~nm}),{ }^{60,61}$ it can be seen that the cyclodimerization of two U monomers leads to a blue shift of the UV absorption maximum. This tendency is also theoretically supported, since for all $\mathrm{CDs}$ of $\mathrm{U}$ we found higher ${ }^{1} \pi \pi^{*}$ vertical excitation energies (more than $6 \mathrm{eV}$ ) than the calculated value of the uracil monomer $(5.54 \mathrm{eV})$. The SDs of $U$ have lower vertical excitation energies of the ${ }^{1} \pi \pi^{*}$ excited state in comparison to the monomer.

The CDs of 6-AU have two low-lying ${ }^{1} \pi \pi^{*}$ excited states. The cis-syn isomer has the lowest vertical excitation energy of the first ${ }^{1} \pi \pi^{*}$ excited state while the trans-syn isomer has the highest. The calculated (CC2/cc-pVDZ) vertical excitation energy of the low-lying ${ }^{1} \pi \pi^{*}$ excited state of the monomer of $6-\mathrm{AU}$ is $5.19 \mathrm{eV}$. This value is higher than the vertical excitation energies of the first bright ${ }^{1} \pi \pi^{*}$ excited state of the CD structures and lower than those of the SDs. Therefore, in contrast to $U$, a red shift of the calculated UV absorption spectra should be expected for 6-AU in the course of the photocyclodimerization. Opposite to the computed data, according to the experimental ${ }^{1} \pi \pi^{\star}$ absorption maximum of 6 -AU monomer $(4.779 \mathrm{eV})$, a blue shift is expected in the course of the cyclodimerizations to cis-anti, trans-syn and trans-anti isomers and no shift for the formation of the cis-syn isomer $(4.774 \mathrm{eV})$.

The SDs of $U$ have lower ${ }^{1} \pi \pi^{*}$ vertical excitation energy than the corresponding CDs. The trans-syn isomer has higher ${ }^{1} \pi \pi^{*}$ vertical excitation energy than cis-anti and trans-anti systems. In the SDs of 6-AU, similar to U, the two low-lying excited states are of ${ }^{1} \mathrm{n} \pi^{*}$ character. In contrast to $\mathrm{U}$, the ${ }^{1} \pi \pi^{*}$ excited-state energy of the cis-syn, cis-anti and trans-anti SDs of 6-AU is higher than that of the corresponding CDs, although that difference is very small in the cis-anti and trans-anti isomers - about $0.076 \mathrm{eV}$ and 0.003 $\mathrm{eV}$, respectively. The trans-syn $\mathrm{SD}$ of 6-AU has higher ${ }^{1} \pi \pi^{*}$ vertical excitation energy than the $\mathrm{CD}$, like $\mathrm{U}$.

Table 1. Vertical excitation energies (CC2) of the SDs and CDs of $\mathrm{U}$ and 6-AU, all in $\mathrm{eV}$. In brackets are given the oscillator strengths

\begin{tabular}{|c|c|c|c|c|c|c|c|c|}
\hline & \multicolumn{8}{|c|}{ CDs } \\
\hline & \multicolumn{2}{|l|}{ cis-syn } & \multicolumn{2}{|c|}{ cis-anti } & \multicolumn{2}{|c|}{ trans-syn } & \multicolumn{2}{|c|}{ trans-anti } \\
\hline \multirow[t]{3}{*}{$\mathrm{U}$} & $5.204(0.0006)$ & ${ }^{1} \mathrm{n} \pi^{*}$ & $5.223(0.0016)$ & ${ }^{1} \mathrm{n} \pi^{*}$ & $5.176(0.0006)$ & ${ }^{1} \mathrm{n} \pi^{*}$ & $5.193(0.0006)$ & ${ }^{1} \mathrm{n} \pi^{*}$ \\
\hline & $5.271(0.0015)$ & ${ }^{1} \mathrm{n} \pi^{*}$ & $5.232(0.0017)$ & ${ }^{1} \mathrm{n} \pi^{*}$ & $5.227(0.00008)$ & ${ }^{1} \mathrm{n} \pi^{*}$ & $5.218(0.0007)$ & ${ }^{1} \mathrm{n} \pi^{*}$ \\
\hline & $6.177(0.0148)$ & ${ }^{1} \pi \pi^{*}$ & $6.207(0.0119)$ & ${ }^{1} \pi \pi^{*}$ & $6.046(0.0710)$ & ${ }^{1} \pi \pi^{*}$ & $6.213(0.0043)$ & ${ }^{1} \pi \pi^{*}$ \\
\hline \multirow[t]{5}{*}{ 6-AU } & $4.774(0.0417)$ & ${ }^{1} \pi \pi^{*}$ & $4.942(0.0166)$ & ${ }^{1} \pi \pi^{*}$ & $5.157(0.0276)$ & ${ }^{1} \pi \pi^{*}$ & $4.929(0.00000001)$ & ${ }^{1} \pi \pi^{*}$ \\
\hline & $4.955(0.0060)$ & ${ }^{1} \pi \pi^{*}$ & $4.996(0.00002)$ & ${ }^{1} \pi \pi^{*}$ & $5.200(0.0239)$ & ${ }^{1} \pi \pi^{*}$ & $5.184(0.0052)$ & ${ }^{1} \mathrm{n} \pi^{*}$ \\
\hline & $5.176(0.0166)$ & ${ }^{1} \mathrm{n} \pi^{*}$ & $5.503(0.0044)$ & ${ }^{1} \mathrm{n} \pi^{*}$ & $5.241(0.00002)$ & ${ }^{1} \mathrm{n} \pi^{*}$ & $5.647(0.00000003)$ & ${ }^{1} \pi \pi^{*}$ \\
\hline & \multicolumn{8}{|c|}{ SDs } \\
\hline & \multicolumn{2}{|l|}{ cis-syn } & \multicolumn{2}{|c|}{ cis-anti } & \multicolumn{2}{|c|}{ trans-syn } & \multicolumn{2}{|c|}{ trans-anti } \\
\hline \multirow[t]{3}{*}{$\mathrm{U}$} & $4.785(0.0002)$ & ${ }^{1} \mathrm{n} \pi^{*}$ & $4.874(0.0001)$ & ${ }^{1} \mathrm{n} \pi^{*}$ & $4.911(0.0002)$ & ${ }^{1} \mathrm{n} \pi^{*}$ & $4.866(0.00005)$ & ${ }^{1} \mathrm{n} \pi^{*}$ \\
\hline & $4.813(0.0002)$ & ${ }^{1} \mathrm{n} \pi^{*}$ & $4.875(0.0006)$ & ${ }^{1} \mathrm{n} \pi^{*}$ & $4.912(0.0001)$ & ${ }^{1} \mathrm{n} \pi^{*}$ & $4.867(0.0005)$ & ${ }^{1} \mathrm{n} \pi^{*}$ \\
\hline & $5.386(0.0883)$ & ${ }^{1} \pi \pi^{*}$ & $5.283(0.0368)$ & ${ }^{1} \pi \pi^{*}$ & $5.426(0.1107)$ & ${ }^{1} \pi \pi^{*}$ & $5.226(0.0004)$ & ${ }^{1} \pi \pi^{*}$ \\
\hline \multirow[t]{3}{*}{ 6-AU } & $4.373(0.0001)$ & ${ }^{1} \mathrm{n} \pi^{*}$ & $4.387(0.0001)$ & ${ }^{1} \mathrm{n} \pi^{*}$ & $4.381(0.0004)$ & ${ }^{1} \mathrm{n} \pi^{*}$ & $4.381(0.0003)$ & ${ }^{1} \mathrm{n} \pi^{*}$ \\
\hline & $4.406(0.0001)$ & ${ }^{1} \mathrm{n} \pi^{*}$ & $4.399(0.0002)$ & ${ }^{1} \mathrm{n} \pi^{*}$ & $4.385(0.000002)$ & ${ }^{1} \mathrm{n} \pi^{*}$ & $4.384(0.000002)$ & ${ }^{1} \mathrm{n} \pi^{*}$ \\
\hline & $5.043(0.0685)$ & ${ }^{1} \pi \pi^{*}$ & $5.015(0.0549)$ & ${ }^{1} \pi \pi^{*}$ & $4.915(0.0040)$ & ${ }^{1} \pi \pi^{*}$ & $4.912(0.00008)$ & ${ }^{1} \pi \pi^{*}$ \\
\hline
\end{tabular}




\section{3. Excited-state Reaction Paths}

In Figs. 4-7 are given the excited-state reaction paths of the proposed gas-phase mechanisms of CD formation of $\mathrm{U}$. The relative energies are referred to the ground-state equilibrium geometries of the SDs, calculated at the CC2/ cc-pVDZ level of theory. In Fig. 4 includes only the cyclodimerization mechanism of cis-syn 6-AU since it has been already investigated for $\mathrm{U}^{40-42}$

The excited-state reaction paths show that after UV-irradiation of the SDs the ${ }^{1} \pi \pi^{*}$ excited states can reach the conical intersections $S_{0} / S_{1}$ without barriers along the excited-state reaction paths. An exception is the trans-syn $(\mathrm{U})$ mechanism where small energy barrier is estimated (Fig. 6). After the conical intersections $\mathrm{S}_{0} / \mathrm{S}_{1}$ the systems can relax non-radiatively (internal conversion) to the ground states $\mathrm{S}_{0}$ of SD or CD. The low-lying ${ }^{1} \pi \pi^{*}$ excited states in the Frank-Condon region of cis-anti (Fig. 5a), trans-syn (Fig. 6a) and trans-anti (Fig. 7a) $\mathrm{CDs}$ of $\mathrm{U}$ have higher energies than those of the corre-

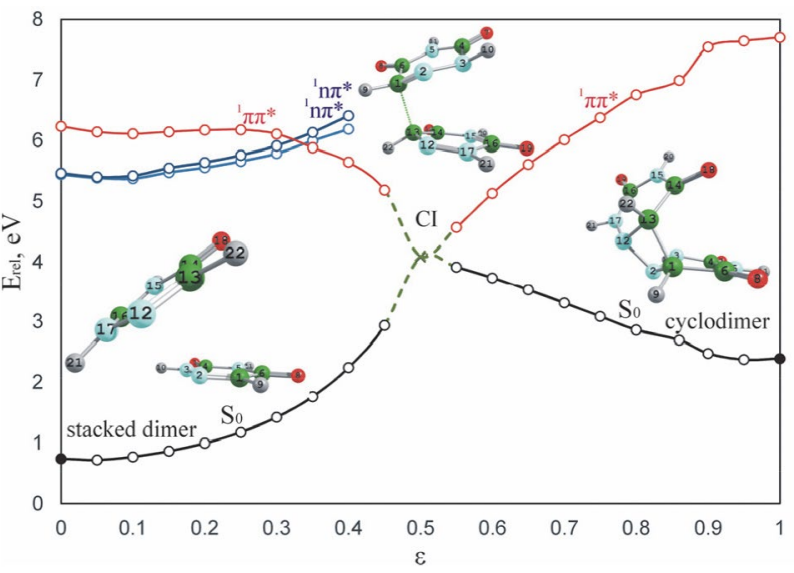

Fig. 4. Excited-state reaction paths (CC2/cc-pVDZ) of the photodimerization of ${ }^{\text {cis-syn }} 6$-AU. The full circles $(\bullet)$ correspond to optimized structures (CASSCF $\left.(2,2) / 6-31 \mathrm{G}^{*}\right)$, while the open circles (O) correspond to linearly-interpolated structures. The conical intersection is designated with $\times$. The relative energy $E_{\text {rel }}$ is referred to the optimized CC2 energy of the SD ( -859.439819 a.u.)
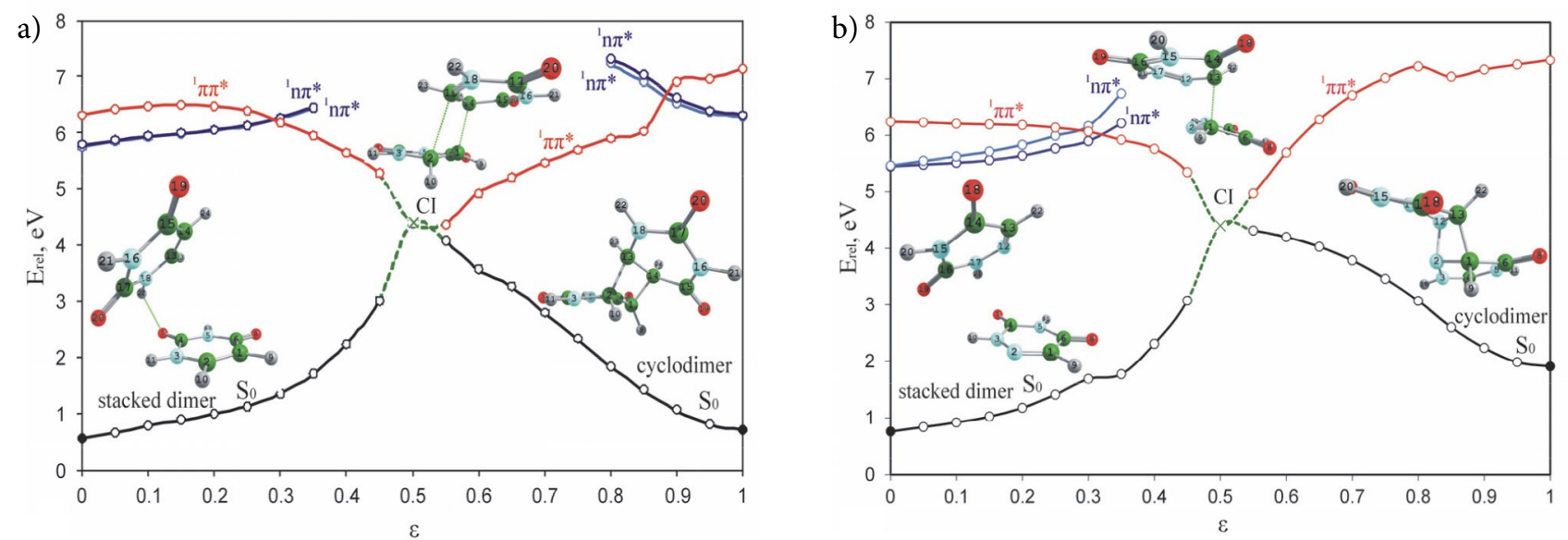

Fig. 5. Excited-state reaction paths (CC2/cc-pVDZ) of the photodimerization of a) ${ }^{\text {cis-anti }} \mathrm{U}$ and b) ${ }^{\text {cis-anti }} 6$-AU. The full circles $(\bullet)$ correspond to optimized structures $\left(\operatorname{CASSCF}(2,2) / 6-31 \mathrm{G}^{*}\right)$, while the open circles $(0)$ correspond to linearly-interpolated structures. The conical intersection is designated with $\times$. The relative energy $\mathrm{E}_{\text {rel }}$ is referred to the optimized CC2 energy of the SD (-827.454581a.u. for U and -859.440401 a.u for 6-AU)

a)

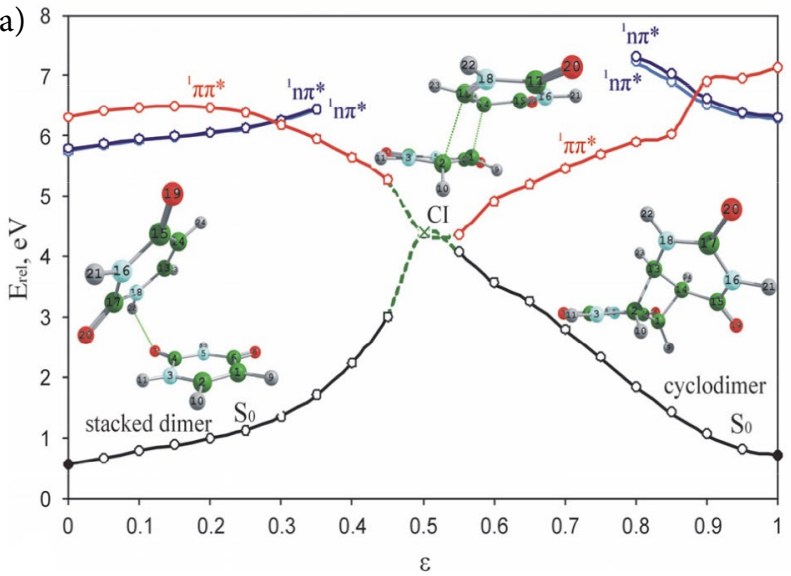

b)

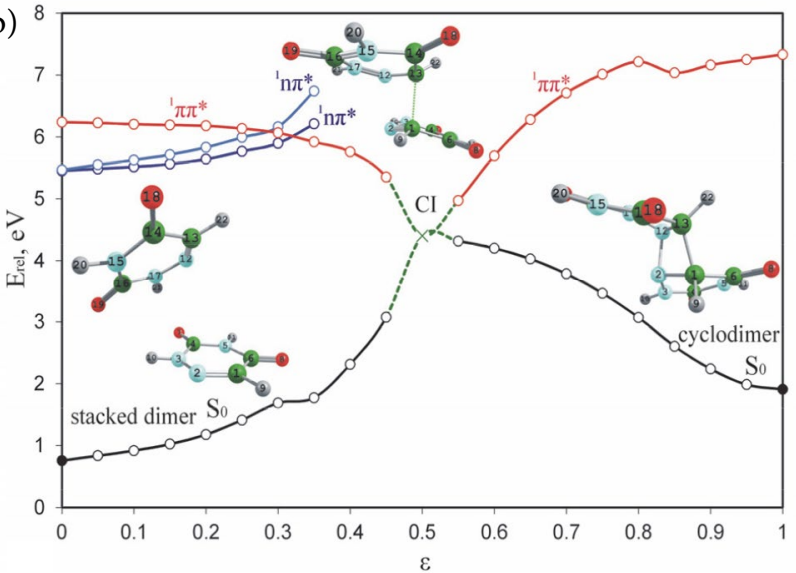

Fig. 6. Excited-state reaction paths (CC2/cc-pVDZ) of the photodimerization of a) ${ }^{\text {trans-syn }} \mathrm{U}$ and b) ${ }^{\text {trans-syn }} 6$-AU. The full circles $(\bullet)$ correspond to optimized structures $\left(\operatorname{CASSCF}(2,2) / 6-31 \mathrm{G}^{*}\right)$, while the open circles $(\mathrm{O})$ correspond to linearly-interpolated structures. The conical intersection is designated with $\times$. The relative energy $\mathrm{E}_{\text {rel }}$ is referred to the optimized CC2 energy of the SD (-827.453428 a.u. for U and -859.439129 a.u for 6-AU) 

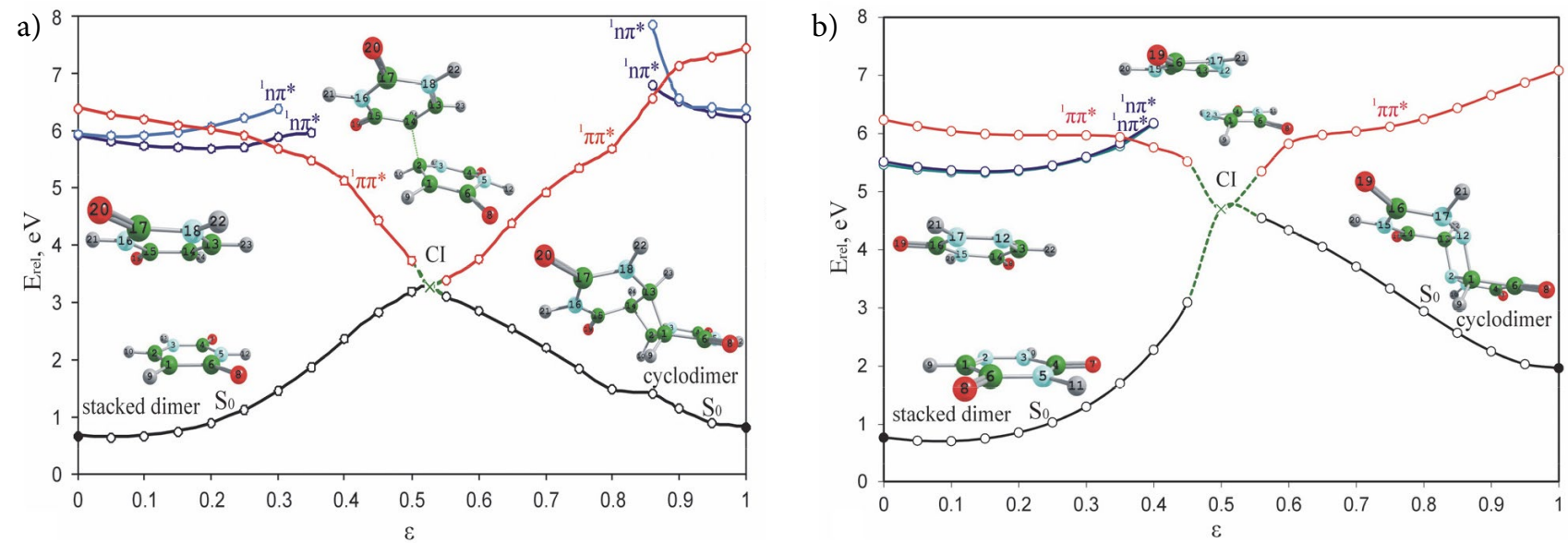

Fig. 7. Excited-state reaction paths (CC2/cc-pVDZ) of the photodimerization of a) trans-anti $U$ and b) ${ }^{\text {trans-anti }} 6-A U$. The full circles $(\bullet)$ correspond to optimized structures $\left(\operatorname{CASSCF}(2,2) / 6-31 \mathrm{G}^{*}\right)$, while the open circles $(\mathrm{O})$ correspond to linearly-interpolated structures. The conical intersection is designated with $\times$. The relative energy $\mathrm{E}_{\text {rel }}$ is referred to the optimized CC2 energy of the SD ( -827.452068 a.u. for $\mathrm{U}$ and -859.438461 a.u for 6-AU)
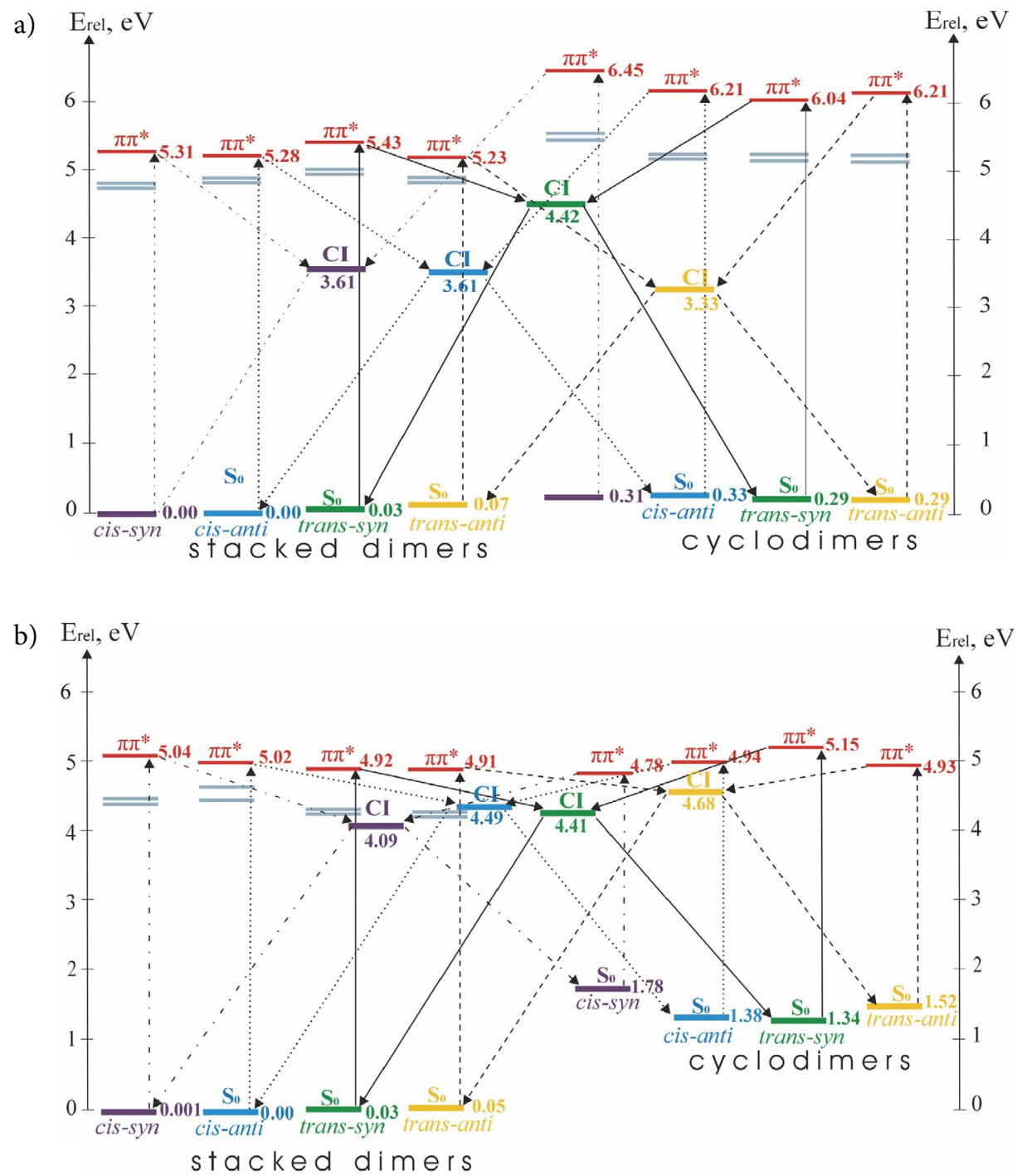

Fig. 8. Energy-level diagrams of the possible relaxation pathways of the ${ }^{1} \pi \pi^{*}$ excited states of the SDs and CDs of a) $U$ and b) 6 -AU. The relative energy $\mathrm{E}_{\text {rel }}$ was referred to the CC2 energy of the optimized cis-anti $\mathrm{SD}(-827.4545801$ a.u. of $\mathrm{U}$ and -859.440401 a.u of 6-AU). The data for $\mathrm{U}$ cis-syn is taken from Ref. [41]. 
sponding SDs. Therefore for the photodestruction of the $\mathrm{CDs}$ of $\mathrm{U}$ by these mechanisms is required higher energy than the energy required for their formation. 6-AU shows opposite effect - for the photodestruction of the $\mathrm{CDs}$ are required lower energies than for their formation.

Conical intersections $S_{2} / S_{1}\left({ }^{1} \pi \pi^{*} /{ }^{1} n \pi^{*}\right)$ are found along all reaction paths of $U$ as well as along the reaction paths of 6-AU starting from the SDs. They are channels through which the excited system can be trapped to the dark ${ }^{1} n \pi^{*}$ excited state. As a result, low intensive fluorescent transitions could be expected as competitive reactions to the cyclodimerizations. On the other hand as competitive mechanisms the reactions can occur through the dark ${ }^{1} n \pi^{*}$ excited states but these reactions will be objects of our special future attention.

All non-radiative mechanisms of photocyclodimerization of $\mathrm{U}$ and 6-AU studied here are summarized in two energy-level diagrams illustrated in Fig. 8. (The data for cis-syn $\mathrm{U}$ is taken from Ref. 41). The driven excited state of the reactions is of ${ }^{1} \pi \pi^{\star}$ type. The excited-state reaction paths of the ${ }^{1} \pi \pi^{*}$ excited states connect the SDs and the CDs through the conical intersections $S_{0} / S_{1}$. The relative energies of the ground states of the SDs and their ${ }^{1} \pi \pi^{*}$ vertical excitation energies are similar for $\mathrm{U}$ and $6-\mathrm{AU}$. The $\mathrm{CDs}$ of $\mathrm{U}$ have lower relative energies of the ground states and higher ${ }^{1} \pi \pi^{*}$ vertical excitation energies than the corresponding structures of 6-AU. All conical intersections $\mathrm{S}_{0} /$ $\mathrm{S}_{1}$ of 6-AU have energies higher than $4 \mathrm{eV}$, similar to the trans-syn isomer of $\mathrm{U}$. The cis-syn, ${ }^{40}$ cis-anti and trans-anti conical intersections $S_{0} / S_{1}$ of $U$ have lower energies which indicates that they could be accessed easily with smaller energy gradient in the course of the reactions in comparison to those of 6-AU.

\section{Conclusion}

The theoretical study (CC2/cc-pVDZ) on the relaxation mechanisms of the photo-induced cyclodimerization reactions of uracil and 6-azauracil to cis-syn, cis-anti, trans-syn and trans-anti cyclodimers proposes gas-phase mechanisms through ${ }^{1} \pi \pi^{*}$ excited-state reaction paths as one of the possible reactions. The mechanisms indicate that the formation of CDs as well as their destruction is a non-radiative reaction passing through conical intersection $\mathrm{S}_{0} / \mathrm{S}_{1}$, which is in accordance with recent studies. ${ }^{40-42}$ The destruction of the CDs of $U$ requires much higher excitation energies than their formation. Opposite to $U$, the formation of the CDs of 6-AU requires higher energies than the energies for their destruction. In other words, according to the proposed mechanisms, the cyclodimerization of two $\mathrm{U}$ molecules to $\mathrm{CD}$ would provoke a blue-shift of the UV absorption maximum, while the formation of $\mathrm{CD}$ of 6 -AU leads to a corresponding red-shift.

\section{Acknowledgment}

The authors thank Prof. Petko Ivanov (Institute of Organic Chemistry, Bulgarian Academy of Sciences) for the computational resource (Linux-cluster MADARA, project RNF01/0110), where the TURBOMOLE computations were performed. We are also thankful to the Department for scientific research at the University of Plovdiv for the financial support (project MU15-HF-012) for the dissemination and popularization of the results.

The investigations are not in conflicts of interest with other researches and do not disturb any ethical rules.

\section{References}

1. A. Downes, Th. P. Blunt, Proc. R. Soc. Lond. 1877, 26, 488-500. DOI:10.1098/rspl.1877.0068

2. L. Serrano-Andrés, M. Merchán in: M. K. Shukla, K. Leszczynski (Eds.): Radiation induced molecular phenomena in nucleic acids, Springer 2008, p. 435.

DOI:10.1007/978-1-4020-8184-2_16

3. M. Merchán, R. Gonzalez-Luque, T. Climent, L. Serrano-Andréz, E. Rodriguez, M. Reguero, D. Pelaez, J. Phys. Chem. B 2006, 110, 26471-26476. DOI:10.1021/jp066874a

4. J. Cadet, E. Sage, T. Douki, Mutat. Res. 2005, 571, 3-17. DOI:10.1016/j.mrfmmm.2004.09.012

5. E. Sage, Photochem. Photobiol. 1993, 57, 163-174. DOI:10.1111/j.1751-1097.1993.tb02273.x

6. G. P. Pfeifer, Photochem. Photobiol. 1997, 65, 270. DOI:10.1111/j.1751-1097.1997.tb08560.x

7. S. Mouret, C. Baudouin, M. Charveron, A. Favier, J. Cadet, T. Douki, Proc. Nat. Acad. Sci 2006, 103, 13765-13770.

DOI:10.1073/pnas.0604213103

8. Y-T. Kao, C. Saxena, L. Wang, A. Sancar, D. Zhong, Proc. Nat. Acad. Sci. 2005, 102, 16128-16132.

DOI:10.1073/pnas.0506586102

9. F. R. de Gruijl, Methods Enzymol. 2000, 319, 359-366. DOI:10.1016/S0076-6879(00)19035-4

10. J. C. van der Leun, F.R. Gruijl de, Photochem. Photobiol. Sci. 2002, 1, 324-326. DOI:10.1039/b201025a

11. Y. Matsumura, H. N. Ananthaswamy, Toxicol. Appl. Pharmacol. 2004, 195, 298-308. DOI:10.1016/j.taap.2003.08.019

12. J. Cadet, P. Vigny in: H. Morrison (ed.): Bioorganic Photochemistry Vol. 1, J. Wiley \& Sons, New York, 1990.

13. T. Douki, J. Cadet, Biochem. (2001), 40, 2495-2501. DOI:10.1021/bi0022543

14. E. C. Friedberg, G. C. Walker, W. Siede, R. D. Wood, R. A. Schultz in: T. Ellenberger (ed): DNA Repair and mutagenesis, ASM Press, Washington, 2006.

15. S. Mouret, C. Phillipe, J. Garcia-Chantegrel, A. Banyasz, S. Karpati, D. Markovitsi, T. Douki, Org. Biomol. Chem. 2010, 8 , 1706-1711. DOI:10.1039/b924712b

16. S. Courdavault, C. Baudouin, M. Charveron, A. Favier, J. Cadet, T. Douki, Mutat. Res. 2004, 556, 135-142.

DOI:10.1016/j.mrfmmm.2004.07.011 
17. S. Kim, S. Jin, G. Pfeifer, Photochem. Photobiol. Sci. 2013, 12, 1409-1415.

Sci. 2013, 12, 1409-1415. DOI:10.1039/c3pp50037c

18. A. Sancar, Biochem. 1994, 33, 2-9.

19. L. Joubert-Doriol, T. Domratcheva, M. Olivucci, A. F. Izmaylov, 2016, arXiv:1602.05044 [physics.chem-ph].

20. E. Adman, L. H. Jensen, Acta Cryst. B 1970, 26, 1326-1334. DOI:10.1107/S0567740870004077

21. A. J. Varghese, Biochem. 1971, 10, 4283-4290.

DOI:10.1021/bi00799a020

22. B. H. Jennings, S. Pastra-Landis, J. W. Lerman, Photochem. Photobiol. 1972, 15, 479-491.

DOI:10.1111/j.1751-1097.1972.tb06259.x

23. M. D. Shetlar, V. J. Basus, Photochemistry and Photobiology 2011, 87, 82-102. DOI:10.1111/j.1751-1097.2010.00826.x

24. E. Adman, M. P. Gordon, L. H. Jensen, Chem. Commun. 1968, 17, 1019-1020. DOI:10.1039/C19680001019

25. C. H. Krauch, D. M. Kramer, P. Chandra, P. Mildner, H. Feller, A. Wacker, Angew. Chem. Int. Ed. Engl. 1967, 6, 956. DOI:10.1002/anie.196709561

26. C. L. Greenstock, H. E. Johns, Biochem. Biophys. Res. Commun. 1968, 30, 21-27. DOI:10.1016/0006-291X(68)90706-7

27. L. A. Frederico, T. A. Kunkel, B.R. Shaw, Biochem 1990, 29, 2532-2537. DOI:10.1021/bi00462a015

28. R. Schinder, A. D. Welch, Science 1957, 125, 548-549. DOI:10.1126/science.125.3247.548

29. B. I. Schinder, E. Frei, J. H. Tuohy, J. Gorman, E. Freireich Jr., J. Clements, Cancer Res. 1960, 20, 28-33.

30. R. E. Handschumacher, A. D. Welch, Cancer Res. 1956, 16, 965.

31. F. Exinger, F. Lacroute, Curr. Genet. 1992, 22, 9-11. DOI:10.1007/BF00351735

32. E. De Clercq, Antiviral Res. 2005, 67, 56-75. DOI:10.1016/j.antiviral.2005.05.001

33. J. Fulara, M. J. Nowak, L. Lapinski, A. Leś, L. Adamowicz, Spetrochim. Acta. 1991, 47, 595-613. DOI:10.1016/0584

34. T. Kobayashi, Y. Harada, T. Suzuki, T. Ichimura, J. Phys. Chem. A 2008, 112, 13308-13315. DOI:10.1021/jp803096j

35. J. P. Gobbo, A. C. Borin, L. Serrano-Andés, J. Phys. Chem. B 2011, 115, 6243-6251. DOI:10.1021/jp200297z

36. M. Etinski, C. Marian, Phys. Chem. Chem. Phys. 2010, 12, 15665-15671. DOI:10.1039/c0cp00106f

37. M. Boggio-Pasqua, G. Groenhof, L. V. Schaefer, H. Grubmueller, M. A. Robb, J. Am. Chem. Soc 2007, 129, 10996-10997. DOI: $10.1021 / \mathrm{ja} 073628 \mathrm{j}$

38. L. Blancafort, A. Migani, J. Am. Chem. Soc 2007, 129, 1454014541 DOI:10.1021/ja074734o

39. J. J. Serrano-Pérez, I. González-Ramírez, P. B. Coto, M. Merchán, L. Serrano-Andréz, J. Phys. Chem. B 2008, 112, 1409614098. DOI:10.1021/jp806794x

40. V. B. Delchev, W. Domke, High performance computing in science and engineering Garching/Munich 2009, 7, 709.
41. I. González-Ramírez, D. Roca-Sanjuán, T. Climent, J. José Serrano-Pérez, M. Merchán, L. Serrano-Andrés, Theor. Chem. Acc. 2011, 128, 705-711. DOI:10.1007/s00214-010-0854-z

42. V. B. Delchev, W. Domcke, J. Photochem. Photobiol A 2013, 271, 1-7. DOI:10.1016/j.jphotochem.2013.07.015

43. D. Roca-Sanjuán, G. Olaso-González, I. González-Ramírez, L. Serrano-Andres, M. Merchan, J. Am. Chem. Soc. 2008, 130, 10768-10779. DOI:10.1021/ja803068n

44. P. Kancheva, V. B. Delchev, J. Mol. Model. 2016, 22, 230. DOI:10.1007/s00894-016-3087-9

45. R. Improta, F. Santoro, L. Blancafort, Chem. Rev. 2016, 116, 3540-93. DOI:10.1021/acs.chemrev.5b00444

46. J. Eisinger, R. G. Shulman, Science 1968, 161, 1311-1319. DOI:10.1126/science.161.3848.1311

47. M. Merchán, L. Serrano-Andréz, M. A. Robb, L. Blancafort, J. Am. Chem. Soc. 2005, 127, 1820-1825. DOI:10.1021/ja044371h

48. L. Liu, B. M. Pilles, J. Gontcharov, D. B. Bucher, W. Zinth, J. Phys. Chem. B 2015, 120, 292-298.

DOI: $10.1021 /$ acs.jpcb.5b08568

49. O. Christiansen, H. Koch, P. Jørgensen, Chem Phys. Lett. 1995, 243, 409-418. DOI:10.1016/0009-2614(95)00841-Q

50. M. J. Frisch et al., GAUSSIAN 03, Gaussian, Inc., Wallingford, CT 2004.

51. R. Ahlrichs, M. Baer, M. Haeser, H. Horn, C. Koelmel, Chem Phys Lett 1989, 162, 165-169.

DOI:10.1016/0009-2614(89)85118-8

52. G. Schaftenar, J. H. Noordik, J. Comput-Aided Mol Design 2000, 14, 123-134. DOI:10.1023/A:1008193805436

53. Chemcraft program 1.6 (built 338), Progranmming: GA Zhurko, Design, additional support: D A Zhurko

54. L. Serrano-Andrés, M. Merchán, J. Photochem. Photobiol. C 2009, 10, 21-32.

55. C. E. Crespo-Hernández, B. Cohen, P. M. Hare, B. Kohler, Chem. Rev. 2004, 104, 1977-2019.

DOI:10.1021/cr0206770

56. K. A. Kistler, S. Matsika, J. Phys. Chem. A 2007,111, 26502661. DOI:10.1021/jp0663661

57. M. Merchán, R. González-Luque, T. Climent, L. Serrano-Andrés, E. Rodríguez, M. Reguero, D. Peláez, J. Phys. Chem. B 2006, 110, 26471-26476. DOI:10.1021/jp066874a

58. Z. Lan, E. Fabiano, W. Thiel, J. Phys. Chem. B 2009, 113, 35483555. DOI:10.1021/jp809085h

59. M. Barbatti, A. J. A. Aquino, J. J. Szymczak, D. Nachtigallová, P. Hobza, Hans Lischka, Proc. Nat. Acad. Sci. 2010, 107, 21453-21458. DOI:10.1073/pnas.1014982107

60. L. B. Clark, G. G. Peschel, I. Tinoco, J. Phys. Chem. 1965, 69, 3615-3618. DOI:10.1021/j100894a063

61. M. K. Shukla, J. Leszczynski, J. Biomol. Struct. Dyn. 2007, 25, 93. DOI:10.1080/07391102.2007.10507159 


\section{Povzetek}

Z uporabo cc-pVDZ baznih funkcij smo na CC2 teorijskem nivoju preučevali fotociklodimerizacijske mehanizme dveh okso tautomerov uracila in 6-azauracila. Uracil smo raziskovali pri treh orientacijah monomerov - cis-anti, trans-sin in trans-anti, medtem ko so bili za 6-azauracil računi izvedeni za vse štiri orientacije. Na CASSCF(2,2)/6-31G* teorijskem nivoju smo ugotovili stožčasta presečišča $S_{0} / S_{1}$ ter predlagali fotofizikalne mehanizme fotoformacije in fotodestrukcije ciklodimerov, ki nastajajo preko ${ }^{1} \pi \pi^{\star}$ elektronsko vzbujenih stan. 